Extreme Adaptive Optics Testbed: High Contrast Measurements with a MEMS Deformable Mirror

J. W. Evans, K. Morzinski, L. Reza, S. Severson, L. Poyneer, B. Macintosh, D. Dillon, G. Sommargren, D.Palmer, D. Gavel, S. Olivier

August 17, 2005

SPIE Optics and Photonics

San Diego, CA, United States

July 31, 2005 through August 4, 2005 
This document was prepared as an account of work sponsored by an agency of the United States Government. Neither the United States Government nor the University of California nor any of their employees, makes any warranty, express or implied, or assumes any legal liability or responsibility for the accuracy, completeness, or usefulness of any information, apparatus, product, or process disclosed, or represents that its use would not infringe privately owned rights. Reference herein to any specific commercial product, process, or service by trade name, trademark, manufacturer, or otherwise, does not necessarily constitute or imply its endorsement, recommendation, or favoring by the United States Government or the University of California. The views and opinions of authors expressed herein do not necessarily state or reflect those of the United States Government or the University of California, and shall not be used for advertising or product endorsement purposes. 


\title{
Extreme Adaptive Optics Testbed: High Contrast Measurements with a MEMS Deformable Mirror
}

\author{
Julia W. Evans ${ }^{1}$, Katie Morzinski ${ }^{2}$, Layra Reza ${ }^{2}$, Scott Severson ${ }^{2}$, Lisa Poyneer ${ }^{1}$, Bruce \\ Macintosh $^{1,2}$, Daren Dillon ${ }^{2}$, Gary Sommargren ${ }^{1}$, David Palmer ${ }^{1,2}$, Don Gavel ${ }^{2}$, Scot Olivier $^{1}$ \\ ${ }^{1}$ Lawrence Livermore National Laboratory, ${ }^{2}$ University of California, Santa Cruz
}

\begin{abstract}
"Extreme" adaptive optics systems are optimized for ultra-high-contrast applications, such as ground-based extrasolar planet detection. The Extreme Adaptive Optics Testbed at UC Santa Cruz is being used to investigate and develop technologies for high-contrast imaging, especially wavefront control. We use a simple optical design to minimize wavefront error and maximize the experimentally achievable contrast. A phase shifting diffraction interferometer (PSDI) measures wavefront errors with sub-nm precision and accuracy for metrology and wavefront control. Previously, we have demonstrated RMS wavefront errors of $<1.5 \mathrm{~nm}$ and a contrast of $>10^{7}$ over a substantial region using a shaped pupil without a deformable mirror. Current work includes the installation and characterization of a 1024actuator Micro-Electro-Mechanical-Systems (MEMS) deformable mirror, manufactured by Boston Micro-Machines for active wavefront control. Using the PSDI as the wavefront sensor we have flattened the deformable mirror to $<1 \mathrm{~nm}$ within the controllable spatial frequencies and measured a contrast in the far field of $>10^{6}$. Consistent flattening required testing and characterization of the individual actuator response, including the effects of dead and low-response actuators. Stability and repeatability of the MEMS devices was also tested. Ultimately this testbed will be used to test all aspects of the system architecture for an extrasolar planet-finding AO system.
\end{abstract}

\section{Introduction}

The field of high contrast imaging has taken on new urgency as astronomers require higher contrasts levels for the imaging of extrasolar planets - potentially one of the most interesting scientific developments of the next decade. A halo of scattered light from the parent star will limit contrast and the ability to image a planet. This halo contains components due to both diffraction and a speckle pattern due to residual phase errors. Using a Taylor series to expand the PSF to second order these components are easily identified ${ }^{1}$. In the equation below, A is the Fourier transform of the aperture and $\Phi$ is the Fourier transform of the phase in the aperture plane. In a system with no phase errors and a circular aperture for example the $\mathrm{PSF}=\mathrm{AA}^{*}$, returns an airy pattern. When phase errors are present the equation is more complex:

$P S F=A A^{*}-i\left[A\left(A^{*} \otimes \Phi^{*}\right)-A^{*}(A \otimes \Phi)\right]+(A \otimes \Phi)\left(A^{*} \otimes \Phi^{*}\right)-\frac{1}{2}\left[A\left(A^{*} \otimes \Phi^{*} \otimes \Phi^{*}\right)+A(A \otimes \Phi \otimes \Phi)\right]$

The first, second and fourth terms in the expansion are all proportional to the perfect diffraction pattern caused by the shape of the aperture. By suppressing diffraction these terms will be controlled. The third term is not proportional to diffraction; rather it is proportional to the phase error in the system. Contrast is reduced by phase error squared.

High-contrast imaging requires that both components be controlled. Wavefront errors can come from both dynamic terms such as residual atmospheric phase (after correction by an adaptive optics system), and quasi-static sources such as internal optical errors and errors in calibration of the adaptive optics system. Atmospheric phase errors produce a PSF halo that becomes smoother with longer integrations, but scattered light caused by static wavefront errors will produce a residual pattern of speckles that will not smooth out over time ${ }^{1}$. Simulations indicate that the contrast level of an Extreme Adaptive Optics system on an 8-10 m telescope would need, to conduct an interesting survey of potential warm Jupiter-like planets, is $10^{-7}$. 
The extreme adaptive optics testbed in the Laboratory for Adaptive Optics at UCSC is designed to test the limits of achievable contrast in the lab and to develop high-contrast technologies for a future extrasolar planet imager. In initial experiments without active wavefront control $6.5 \cdot 10^{-8}$ was demonstrated with a total RMS wavefront error of $\sim 1.5$ $\mathrm{nm}^{3}$. A 1024-actuator MEMS deformable mirror has been added to the system. Characterization of un-powered flatness, actuator yield and voltage response have been completed for 8 devices. Closed loop performance, and the corresponding contrast in the far field have been measured. A goal of contrast measurements $>10^{-6}$ and wavefront error of $<1 \mathrm{~nm}$ in the control band were set for MEMS performance.

\section{Experimental Method}

The ExAO testbed was designed with minimal, high-quality optics to ensure end to end wavefront error would be small $(<1.5 \mathrm{~nm}$ RMS). In initial high contrast measurements active wavefront control was not required, allowing early experiments to focus on, suppressing diffraction, understanding the high contrast regime and developing experimental methods for high contrast imaging (i.e. controlling scattered light). In the current set-up a 1024-actuator MEMS deformable mirror, developed by Boston Micro-Machines, is used for wavefront control. The testbed has two modes of operation: far-field imaging, and wavefront measurement interferometry. The testbed consists of a laser source (532 nm) passed through an optical fiber and a high quality $(<1 \mathrm{~nm}$ RMS) lens. The beam passes through a pupil stop, reflects off the flat or DM. In imaging mode, the beam is then imaged onto a CCD sampled at $\sim 5$ times the Nyquist limit (see Figure 1). Although there is no re-imaged focal/pupil plane for a Lyot coronagraph, diffraction is suppressed by apodizing the pupil with a prolate shaped pupil ${ }^{4,5}$. Simple shaped pupils only suppress diffraction over a narrow range of angles, which limits their utility for astronomical operations, but produces a region of high contrast sufficient for laboratory testing and has the advantage of not introducing additional phase aberrations.

In Phase Shifting Diffraction Interferometer (PSDI) mode, the testbed becomes an extremely accurate $(<0.5 \mathrm{~nm}$ RMS absolute wavefront accuracy) optical metrology system. The PSDI was developed at LLNL for metrology of complex aspheric optics ${ }^{6}$. Briefly, a probe wavefront is injected from the upper single-mode fiber in Figure 1.

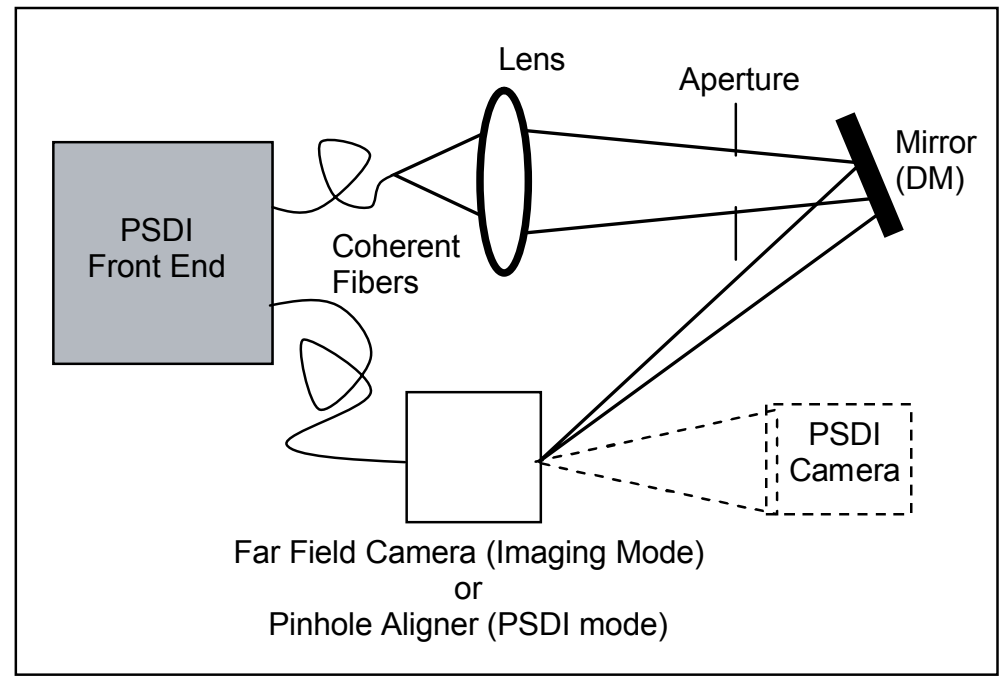

Figure 1: Simplified version of the ExAO testbed Layout. This passes through the system and is focused onto a pinhole embedded in a super polished flat mirror (the pinhole aligner). Meanwhile, a coherent reference beam passes through the pinhole and interferes with the outgoing probe wavefront. The interference pattern is recorded at a CCD located in an arbitrary location in free space. Using standard phase-shifting interferometer techniques this produces a measurement of the wavefront at this location, which can then be numerically propagated to the plane of interest, such as the MEMS plane of the system. Wavefront measurements indicate if the system is meeting our goals for wavefront quality and provide a consistency check for the contrast measurements. The resolution of the PSDI is limited by truncation effects due to an aperture at the pinhole aligner. The effective resolution in the MEMS plane is $\sim 141 \mu \mathrm{m}$.

For closed loop operation, programs in IDL are used to direct data acquisition and back propagation of the PSDI and to command the MEMS device through the MEMS driver. Before closed loop operation the alignment and voltage 
response of the system must be calibrated. Current operations use the average voltage response of four actuators for the entire device, but recently the ability to calibrate individual actuators has been added. The MEMS is controlled with 13bit Red Nun electronics. The smallest voltage step allowed with these electronics is 0.025 volts. When a 3 by 3 array of actuators is moved with a zero volt bias level the stroke of the device is approximately $1.2 \mu \mathrm{m}$. Moving an individual actuator, or operating the device at a bias level, slightly reduces the stroke of the device.

\section{Results and Discussion}

\subsection{Closed Loop Performance and Limitations}

During closed loop operation a 10-mm circular aperture is placed slightly in front of the MEMS device giving an effective aperture of $9.2 \mathrm{~mm}$. Approximately 28 actuators are across the aperture, yielding a highest controllable spatial frequency of 14 cycles per aperture and a corresponding control radius in the point spread function of $14 \lambda / \mathrm{D}$. A ring of two actuators around the edge of the aperture are slaved to the final ring of actuators inside the aperture to reduce edge effects using a nearest neighbor average. Typically the closed loop is run for between 12-15 iterations, and most of the correction takes place within the first five iterations. Tip/Tilt is not actively controlled; it is removed by hand after several iterations and numerically thereafter. Before running closed loop the wavefront has $109.6 \mathrm{~nm}$ total RMS wavefront error, and after flattening the wavefront has $6.8 \mathrm{~nm}$ total RMS wavefront error with $1.2 \mathrm{~nm}$ inside the controlled range of spatial frequencies (See Figure 2).

The wavefront error is dominated by structures at the scale of an individual actuator. By numerically filtering out spatial frequencies beyond the highest controllable spatial frequency, the effect of irregular actuators on the flattened shape is revealed (See Figure 3). There are two irregular actuators near the edge of the aperture and another in the center. The edge of the aperture may be contaminated by these irregular actuators, the slaved actuators outside of the aperture or a measurement effect. Calculating the RMS over a slightly smaller aperture $(8.75 \mathrm{~mm})$ the RMS wavefront error is reduced
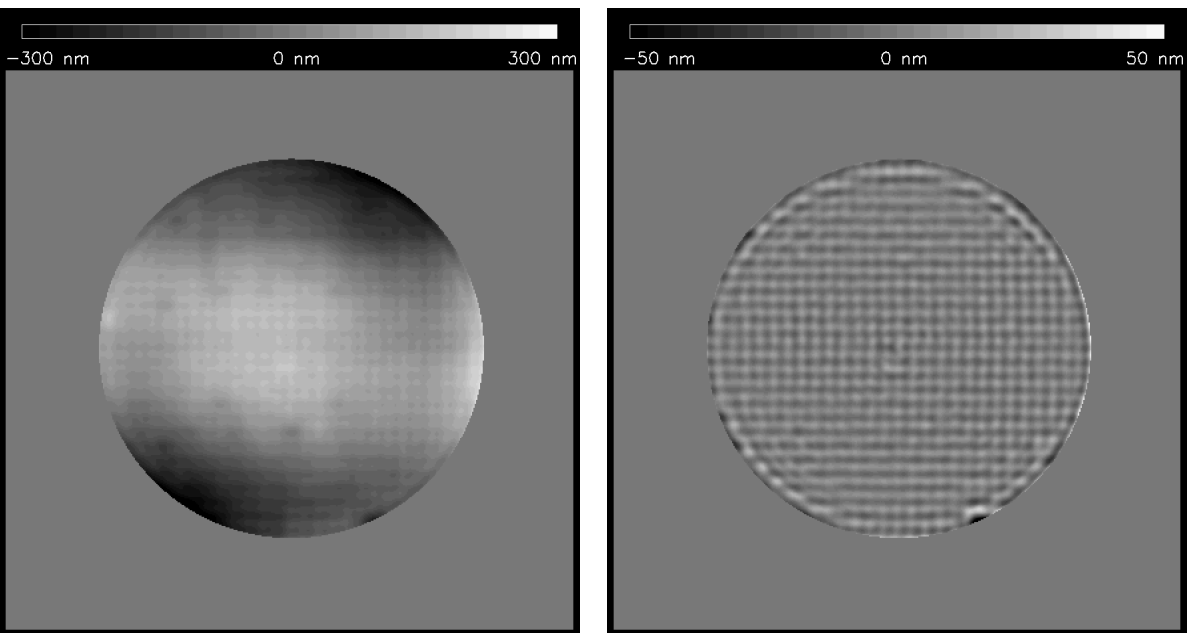

Figure 2: Wavefront measurements from before and after closed loop operation. The flattened wavefront is dominated by structures at the scale of an individual actuator. to $0.93 \mathrm{~nm}$ inside the control band, meeting the goal of $<1 \mathrm{~nm}$ set for this work. In a small aperture chosen to avoid any irregular actuators the RMS wavefront error is reduced to $0.68 \mathrm{~nm}$ inside the control band. Clearly any irregular actuators even partially inside the aperture will limit performance. 
Three other potential limitations to improved closed loop performance have been investigated: stability, measurement error and voltage step size. To test MEMS stability a flattened shape is applied to the MEMS device and successive wavefront measurements are taken every $38 \mathrm{~s}$ (the minimum time to complete a PSDI measurement) for 60 iterations. The variation of each actuator from its initial position is calculated with piston and tip/tilt removed (see figure). This analysis was done over approximately one third of the device (367 actuators). Of the actuators tested $96 \%$ have a standard deviation from their original position of $<0.3 \mathrm{~nm}$. A deviation of $<0.2 \mathrm{~nm}$ is achieved by $89 \%$ of actuators and $62 \%$ deviate $<0.1 \mathrm{~nm}$. It is interesting to note the direct correlation between irregular actuators discovered during closed loop and unstable actuators. Not only are many of these irregular actuators also unstable but nearby actuators not identified during closed loop operation may also be unstable. The effect of irregular actuators on stability further indicates that irregular actuators limit closed loop performance more than anything else. The median standard deviation of all 367 measured actuators is $0.15 \mathrm{~nm}$. The stability test was also run with a flat mirror replacing the MEMS and the equivalent standard deviation per "actuator" was $0.12 \mathrm{~nm}$. This indicates that most of the actuators on the MEMS are stable within our measurement limit. Techniques for further reducing measurement error are being investigated. The

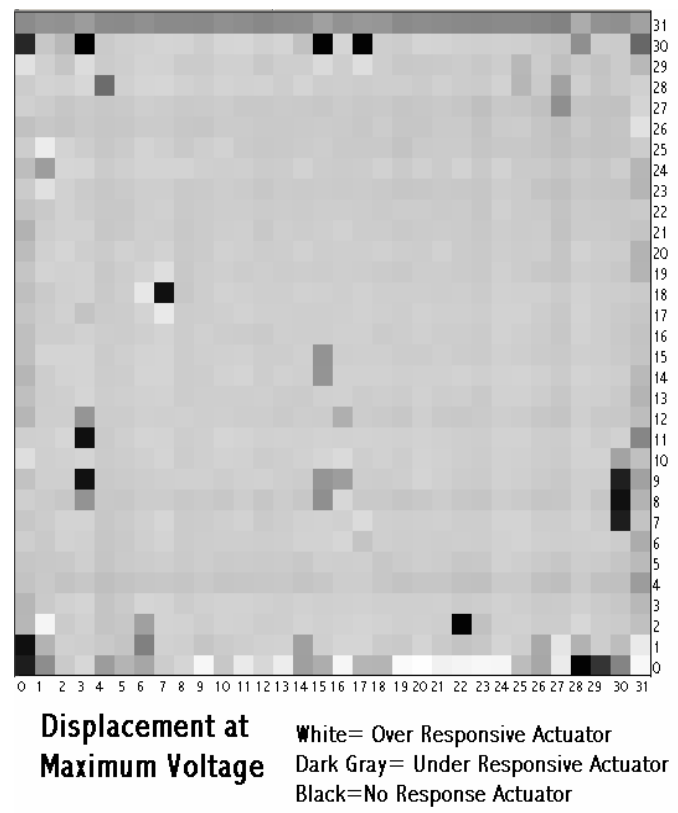

Figure 4: The maximum of displacement of all actuators on a single MEMS device were measured individually and plotted above. Most of the actuators vary less than $2.6 \%$

\begin{tabular}{|c|c|c|}
\hline $\begin{array}{c}\text { Aperture } \\
(\mathrm{mm})\end{array}$ & $\begin{array}{c}\text { Total RMS WFE } \\
(\mathrm{nm})\end{array}$ & $\begin{array}{c}\text { In Band RMS WFE } \\
(\mathrm{nm})\end{array}$ \\
\hline 9.2 & 6.75 & 1.19 \\
\hline 8.75 & 6.06 & 0.93 \\
\hline 7.0 & 5.74 & 0.72 \\
\hline 3.0 & 5.39 & 0.68 \\
\hline
\end{tabular}

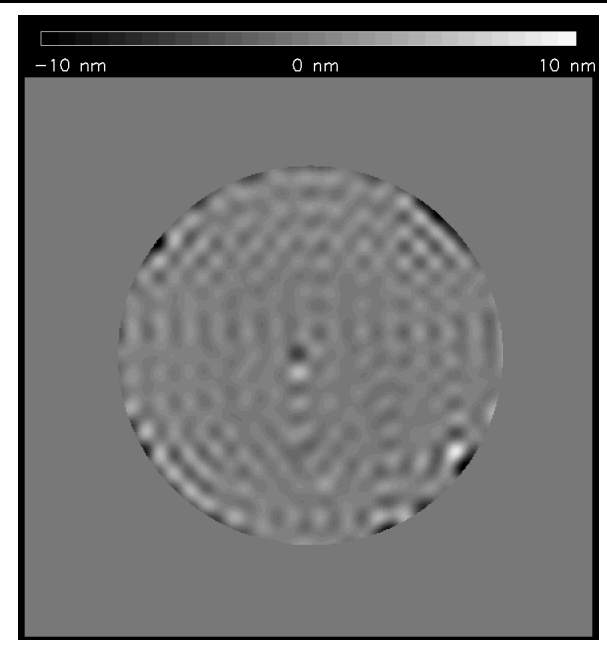

Figure 3: The table above lists the RMS wavefront error for various size apertures, beginning with the flattened wavefront shown.

last potential error source that has been investigated is the smallest voltage step that can be applied by the 13-bit electronics. The value for the step size was calculated and measured to be 0.025 volts. Depending on the bias level used for operation this correspond to phase error of 0.12 $0.18 \mathrm{~nm}$, indicating that the closed loop system cannot flatten better than this value.

\subsection{MEMS Characterization}

\subsubsection{Irregular actuators}

MEMS actuators have a non-linear response that varies from device to device, and some actuators have even more irregular responses. We measured the response of every actuator on a single MEMS device. Based on that test and 
experience with other devices irregular actuators can be broken down into three categories: no displacement, low response and high response. Figure 4 shows the maximum displacement of every actuator measured individually. Most of the actuators are the median gray color indicating a normal voltage response. Those actuators have quadratic voltage responses that vary less than $2.6 \%$. The black actuators have no displacement, which can indicate a completely dead actuator or an actuator that only moves under the influence of neighboring actuators. Under-responding actuators were initially thought to have shallower voltage response curves; however this test indicates that most low response actuators have their motion clipped to no greater displacement than their neighbors.

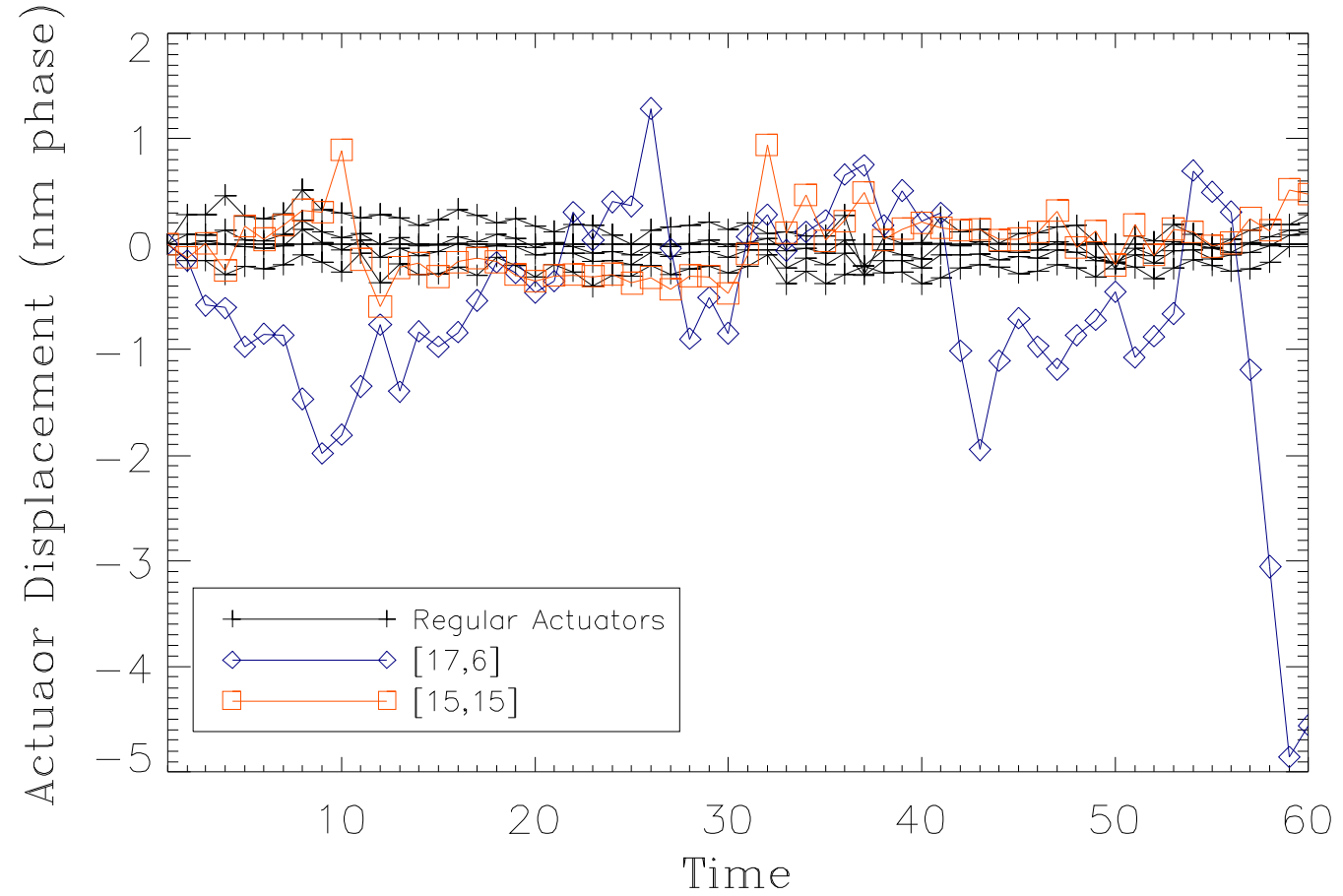

Figure 4: Stability measurements were made by applying a flattened shape to the device and taking a wavefront measurement every $38 \mathrm{~s}$ for 38 minutes. Most of the actuators are more stable than our ability to measure.

\subsubsection{Actuator repeatability}

The ability of a MEMS actuator to return to a previous voltage position is not important in closed loop operation. Actuator repeatability is primarily important for open loop operations. However from a practical stand point it is quicker to re-apply a previous flattening than to re-run the closed loop procedure. The 367 actuators that were evaluated during stability testing were also tested for actuator repeatability. As with the stability test most actuators have repeatable positioning, the same group of irregular actuators identified during closed loop and stability tests, and some neighboring actuators, are also not repeatable. For this test the voltage for a flattening was reapplied before each measurement and in between measurements the device was pushed to the 160 volt position or the 0 volt position, alternately, over a 20 minute period. The standard deviation from the original position of $97 \%$ of the evaluated actuators was $1.0 \mathrm{~nm}$ and $73 \%$ had a deviation of less than $0.4 \mathrm{~nm}$. 


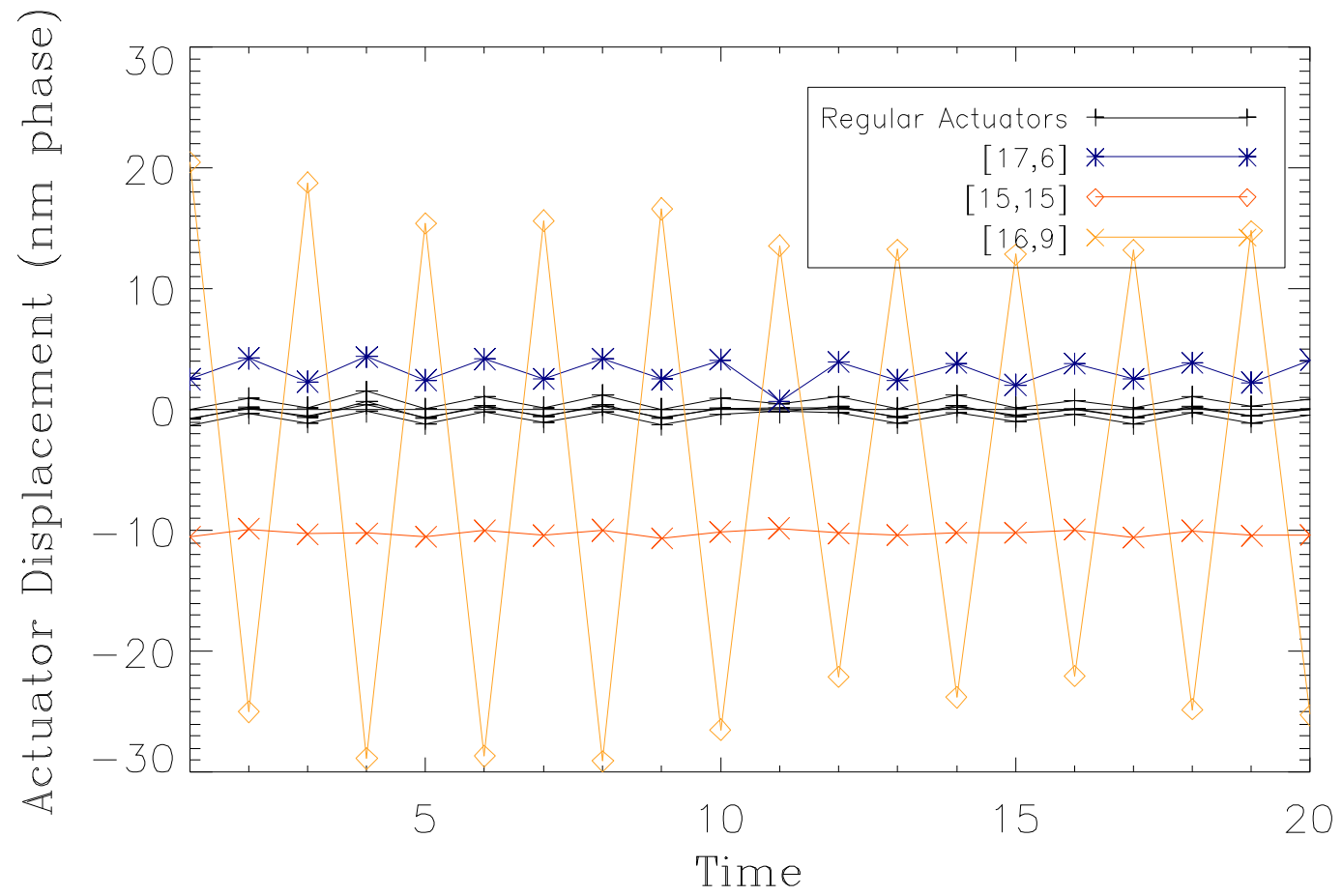

Figure 5: For the actuator repeatability test the voltage for a flattening was reapplied before each measurement and in between measurements the device was pushed to the 160 volt position or the 0 volt position, alternately, over a 20 minute period.

\subsection{High Contrast Measurements}

Contrast measurements of a system can be made directly by comparing the intensity of the core and wings in the far field image. There are several instrumental effects that limit achievable contrast in high-contrast imaging: insufficient dynamic range, scattered light from the optical system, and CCD saturation effects. The imaging technique used in measurements with the flat mirror to minimize these error sources was also used for the MEMS results. Typically an unsaturated short-exposure image using neutral density filters and a highly exposed image in which the PSF core is saturated are used. For analysis each data set of 10 frames is averaged and the corresponding averaged dark frame is subtracted. Then each image is scaled according to the measured attenuation from the ND and integration time to be comparable with the saturated image. All of the images are normalized to the peak of the scaled unsaturated image. Figure 7 is a composite of radial averages from three images. The images are averaged over 4 degrees from the center of the unsaturated core. In the region from $7-14 \lambda / \mathrm{D}$ the radial average contrast is $7.8 \cdot 10^{-7}$. A substantial region in the PSF has $>10^{-6}$ contrast, meeting the specifications for current generation ground based planet imager. Behind the focal plane mask the noise floor is evident at below $5 \cdot 10^{-8}$, indicating that the system is not noise limited. For these measurements a prolate pupil the size of the aperture used for closed loop operation was used to suppress diffraction. A similar experiment with the flat mirror indicates that contrast measurements with the MEMS device are limited by wavefront error. 


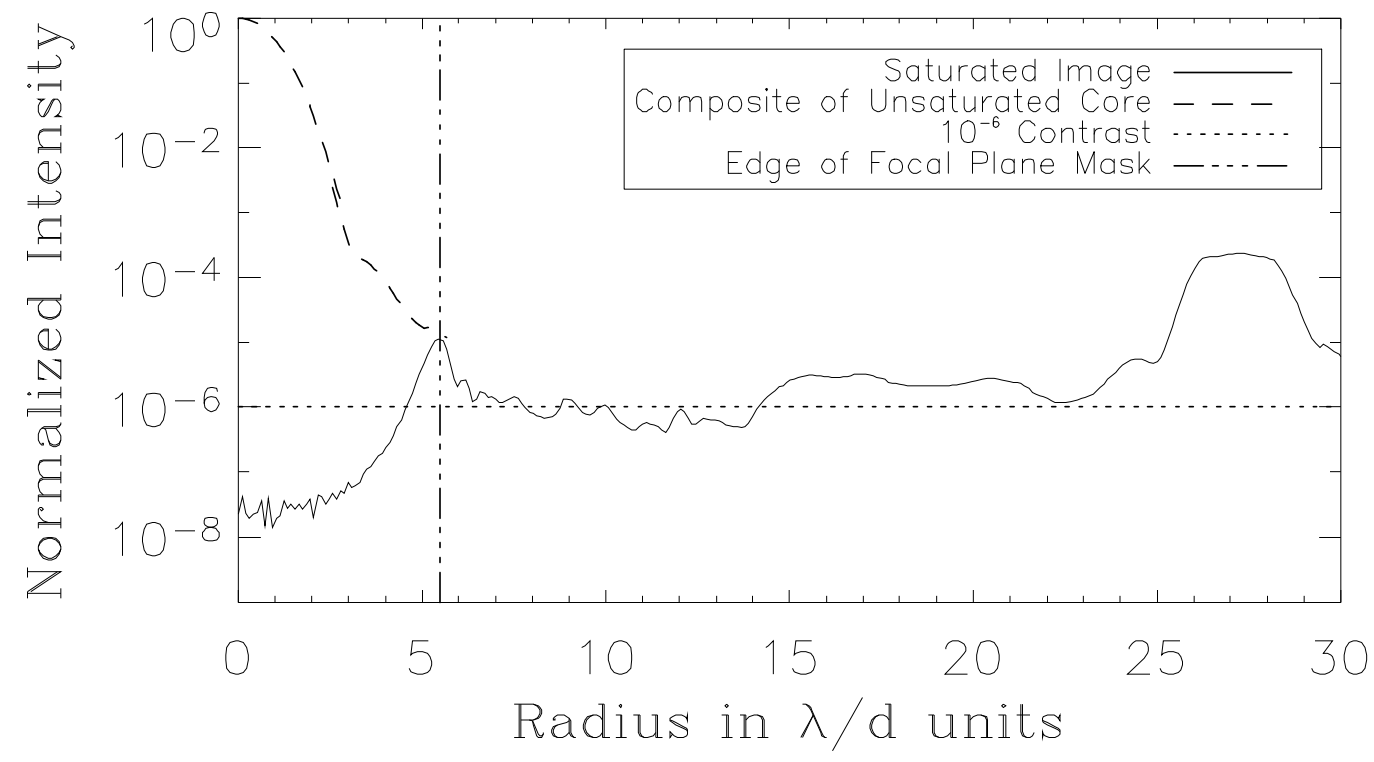

Figure 7: A radial averaged lineout over 4 degrees in the high contrast region of the far field. The average from $7-14 \lambda / \mathrm{D}$ is $7.8 \cdot 10^{-7}$. The edge of the region is set by the half the number of actuators across the aperture (14).

\section{Performance with Additional Phase Aberrations}

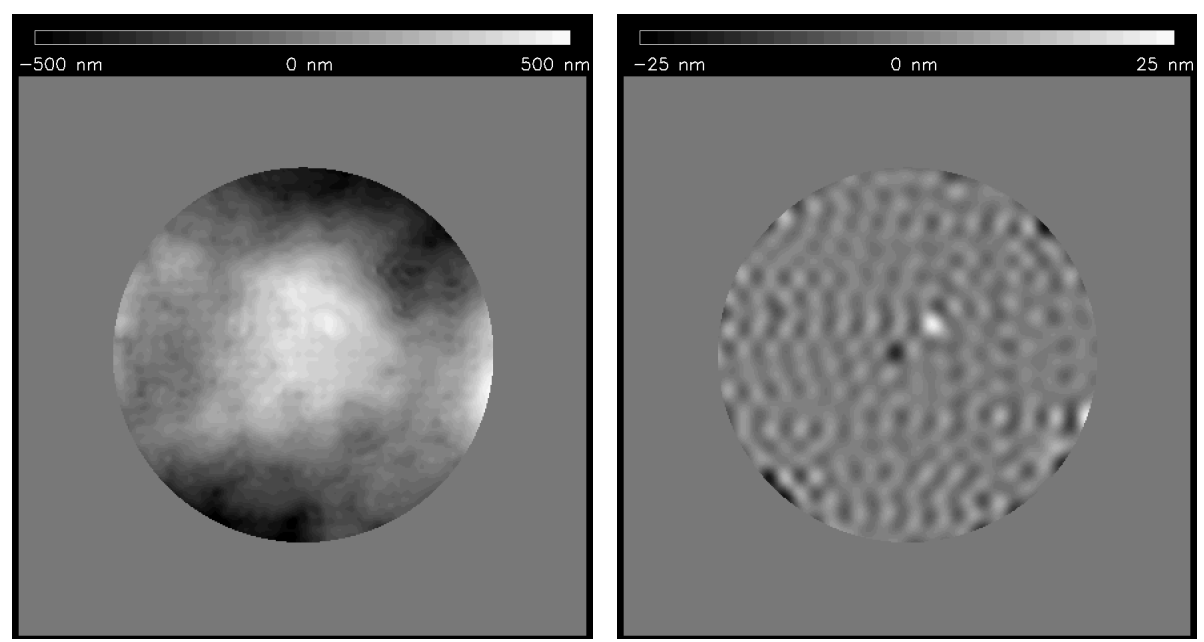

Figure 8: An etched glass phase plate with a discrete approximation to Kolmogorov statistics was introduced and close loop was run. The final wavefront has an RMS of $3.0 \mathrm{~nm}$ within controllable spatial frequencies.

In all previous data with the MEMS device the largest source of phase aberration in the system was the device itself. In any practical system that will not be the case, making testing with an additional aberration an obvious next step. A glass etched phase screen with a discrete approximation to Kolmogorov statistics was introduced directly in front of the aperture of the system. Before flattening the system (MEMS and phase screen) has a total RMS wavefront error of $208.3 \mathrm{~nm}$. After running closed loop, the RMS wavefront error is $14.6 \mathrm{~nm}$, with $3.0 \mathrm{~nm}$ within the controlled spatial 
frequency range. The limitations to closed loop performance with the phase screen have not been investigated, but a filtered wavefront indicates that irregular actuators are still a factor. Corresponding far field images can be taken with the phase screen as well. With $3.0 \mathrm{~nm}$ of wavefront error, the radial averaged contrast from 7-14 $\lambda / \mathrm{D}$ is reduced to 5.53 . $10^{-5}$.

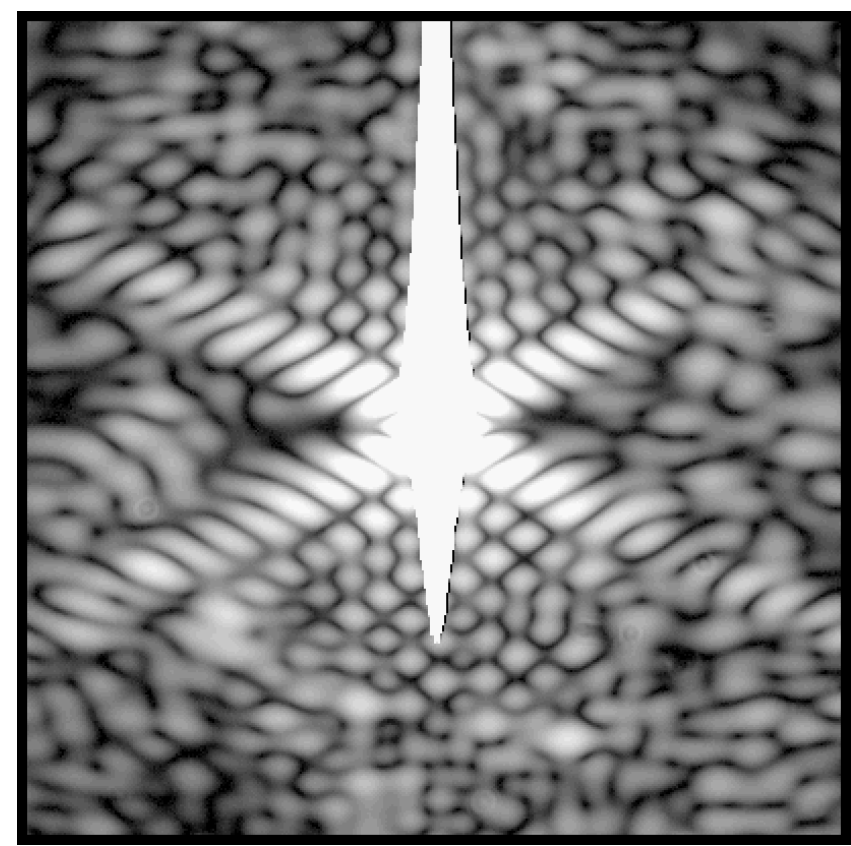

Figure 9: A saturated far field image with the characteristic diffraction pattern produced by prolate spheroid shaped pupil. Residual phase errors from the phase screen produce speckles and limit contrast.

\section{Conclusions and Future Work}

The ExAO testbed has demonstrate the necessary flattening of a DM and the corresponding contrast required for a ground based young Jupiter planet imager. The techniques for characterizing MEMS devices have been improved and may lead to improved performance. We have found that irregular actuators limit the closed loop performance, stability and repeatability of the device. Closed loop tests with additional aberrators are ongoing.

\section{ACKNOWLEDGEMENTS}

This research was performed under the auspices of the U.S. Department of Energy by the University of California, Lawrence Livermore National Laboratory under Contract W-7405-ENG-48, and also supported in part by the National Science Foundation Science and Technology Center for Adaptive Optics, managed by the University of California at Santa Cruz under cooperative agreement No. AST - 9876783. Support for this work was also provided by a grant from the Gordon and Betty Moore Foundation to the Regents of the University of California, Santa Cruz, on behalf of the Laboratory for Adaptive Optics. The content of the information does not necessarily reflect the position or the policy of the Gordon and Betty Moore Foundation, and no official endorsement should be inferred.

\section{References}

1. Sivaramakrishnan, A., J. P. Lloyd, P. E. Hodge et al., Astrophysical Journal 581 (1), L59 2002.

2. Burrows, A. et al. 1997, Ap.J., 491, 856

3. Wilhelmsen Evans, J., Sommargren, G., Poyneer, L., Macintosh, B., Severson, S., Dillon, D., Sheinis, A., Palmer, D., Kasdin, J., Olivier, S.,’Extreme Adaptive Optics Testbed: Results and Future Work', 2004, Proc. SPIE 5490, 954.

4. Jacquinot, P. and B. Roizen-Dossier, in Progress in Optics (North-Holland Publishing Company, Amsterdam, 1964), Vol. 3, pp. 31.

5. Kasdin, N. J., R. J. Vanderbei, D. N. Spergel et al., Astrophysical Journal 582 (2), 11472003.

6. Sommargren, G., D. Phillion, M. Johnson et al., presented at the Emerging Lithographic Technologies VI, 2002. 